\title{
RECLAMACIONES DE PACIENTES EN EL SERVICIO DE EMERGENCIA ADULTOS DE UN HOSPITAL DE TERCER NIVEL DE ATENCIÓN
}

\author{
PATIENT COMPLAINTS IN THE ADULT EMERGENCY DEPARTMENT \\ OF A TERTIARY REFERRAL HOSPITAL \\ Isabel J. Alamo-Palomino', Juan P. Matzumura-Kasano ${ }^{2,3}$, Hugo F. Gutiérrez-Crespo²
}

\begin{abstract}
RESUMEN
Introducción: La condición crítica del paciente que acude a emergencia genera la necesidad de otorgar una atención oportuna, segura y cálida. No cubrir estas expectativas desencadena en la insatisfacción del paciente, reflejada mediante reclamaciones, las cuales en su mayoría resultan justificadas, generando oportunidades de mejora y medidas correctivas. Objetivo: Analizar las reclamaciones presentadas por el paciente y sus familiares en el servicio de emergencia adultos del Hospital Nacional Edgardo Rebagliati Martins. Métodos: Diseño no experimental, descriptivo, de corte transversal. La muestra estuvo conformada por 109 reclamaciones durante el periodo comprendido de mayo a noviembre 2019. Se utilizó una ficha de recolección de datos con la información del Libro de Reclamaciones en Salud. Resultados: Se identificaron 187 motivos de reclamaciones. Los resultados mostraron que 47,7\% fueron presentadas por familiares directos y que $43 \%$ correspondieron al área de medicina interna. En la dimensión inadecuado trato por parte del personal asistencial, $13,9 \%$ de pacientes mostraron disconformidad frente al trato descortés y grosero; en la dimensión demora en la atención, 12,8\% manifestaron encontrarse descontentos por la falta de atención oportuna y, en la dimensión desacuerdo del usuario con la atención o tratamiento recibido, $10,2 \%$ quedaron insatisfechos con la atención recibida. $60,6 \%$ de reclamaciones se resolvieron satisfactoriamente dentro del plazo exigido en la norma. Conclusión: La mayor cantidad de reclamaciones correspondieron al tipo prestacional, asociadas a la atención directa del paciente.
\end{abstract}

Palabras clave: Paciente; Emergencia; Atención (fuente: DeCS BIREME).

\begin{abstract}
Introduction: The critical condition of the patient who presents to an emergency department creates a need to provide timely, safe and warm care. Failing to meet these expectations causes patient dissatisfaction, reflected by complaints, which are mostly justified and lead to opportunities for improvement and corrective measures. Objective: To analyze complaints presented by patients and their relatives at the Adult Emergency Department of the Edgardo Rebagliati Martins National Hospital. Methods: Nonexperimental, descriptive, cross-sectional study. Our sample was comprised of 109 complaints between May and November 2019. We used a data collection sheet based on the information in the complaints book for health care services. Results: We identified 187 reasons for complaints. Our results show that direct relatives filed 47.7\%; 43\% involved Internal Medicine consultations. In the dimension "inadequate treatment by healthcare workers," $13.9 \%$ of patients complained about impolite and rude treatment. In the dimension "delay in care", $12.8 \%$ were discontent due to the lack of timely care. In the dimension "user disagreement with care or treatment received," $10.2 \%$ were dissatisfied with the care they were provided. $60.6 \%$ of complaints were satisfactorily resolved within the period specified by current regulations. Conclusion: Most complaints were related to the direct care of the patient.
\end{abstract}

Key words: Patient; Emergencies; Attention Delivery of Health (source: MeSH NLM).

${ }^{1}$ Oficina de Calidad, Red Prestacional Rebagliati - ESSALUD, Lima-Perú

${ }^{2}$ Facultad de Medicina de la Universidad Nacional Mayor de San Marcos, Lima-Perú.

${ }^{3}$ Posgrado e investigación de la Universidad Nacional Mayor de San Marcos, Lima-Perú.

Citar como: Isabel J. Alamo-Palomino, Juan P. Matzumura-Kasano, Hugo F. Gutiérrez-Crespo. Reclamaciones de pacientes en el servicio de emergencia adultos de un hospital de tercer nivel de atención. Rev. Fac. Med. Hum. Abril 2020; 20(2):246-253. DOI 10.25176/RFMH.v20i2.2916 


\section{INTRODUCCIÓN}

El servicio de emergencia es el área encargada de brindar una atención oportuna y segura a los pacientes que acuden por una atención médica de urgencia debido al problema crítico de salud que presentan. En esta área laboran diferentes tipos de profesionales y acuden un gran número de pacientes. En los últimos años, el incremento de la demanda y el crecimiento deficiente de la oferta ocasionan que los pacientes pasen por largos tiempos de espera para recibir la atención solicitada en dichos servicios, generándose situaciones de estrés, sobrecarga laboral en los profesionales de la salud, demora en la atención, posibles malos resultados en el proceso de atención e insatisfacción de los pacientes ${ }^{(1)}$. Acudir a los servicios de emergencias resulta también estresante para el paciente y sus familiares, desencadena un desequilibro en el núcleo familiar, afectándolos a nivel emocional, lo cual puede alterar su comportamiento y su percepción al momento de afrontar una situación de emergencia(2).

Resulta importante enfatizar que los servicios de emergencia garantizan uno de los derechos fundamentales de la población; de otro lado, tratar de lograr el aseguramiento universal en salud ha generado mayor interés por mejorar la calidad en la atención ${ }^{(3)}$. Conocer las expectativas del paciente que acude a recibir la prestación de salud, permite al establecimiento comprender las necesidades y brindar una atención de calidad, teniendo en cuenta que una mala calidad en la atención se encuentra asociada a insatisfacción, la cual produce una ruptura en la relación médico paciente, genera una experiencia negativa en el paciente que puede ser transmitida a familiares, dañando la imagen del establecimiento o expresarse a través de reclamaciones ${ }^{(4,5,6)}$. En ese sentido, la perspectiva del paciente es importante, debido a su sensibilidad y capacidad para reconocer una amplia gama de problemas de las atenciones de salud recibidas, por la visión diferente que tienen de los problemas frente a los reportados por el personal de salud. Por tanto, las reclamaciones proporcionan una importante información adicional para la organización de salud sobre cómo mejorar los procesos de atención ${ }^{(7,8)}$.

Los pacientes que acuden a los servicios de emergencia tienen la necesidad de recibir una información clara, congruente y completa, relacionada al problema de salud que los motivó a acudir, a absolver sus dudas o preocupaciones en relación al tratamiento, procedimiento diagnóstico o terapéutica a los cuales son o serán sometidos, a recibir una atención rápida, oportuna, segura y de calidad, a recibir en todo momento un trato cálido y humano, a encontrarse en ambientes óptimos que cuenten con las condiciones necesarias de infraestructura, equipamiento, materiales y medicamentos que aseguren la atención ${ }^{(9)}$. Cuando estas condiciones no se dan, surgen reclamaciones, que constituyen indicadores fundamentales de los problemas en el sistema de salud; por estas razones, hoy en día son reconocidas como una fuente de información valiosa del proceso de atención y pueden utilizarse de manera positiva para reflexionar sobre la práctica y los servicios que se brindan, permitiendo establecer oportunidades de mejora(10,11).

El análisis de reclamaciones permite reflexionar y reconocer problemas en la seguridad del paciente, puede utilizarse de manera efectiva para identificar eventos adversos mediante los cuales los servicios involucrados desarrollen e implementen planes de mejora para optimizar la calidad de la atención ${ }^{(12)}$. Sin embargo, es importante enfatizar que las reclamaciones no cuentan con una clasificación estándar, por el contrario, se clasifican de diferente manera, en diferentes sistemas de salud y en diferentes países, lo que genera problemas al momento de realizar comparaciones y extraer conclusiones, dificultando el desarrollo de un enfoque como política nacional ${ }^{(13)}$. Por lo descrito, se han iniciado investigaciones para examinar sistemáticamente las reclamaciones del paciente y sus familiares, para comprender y mejorar las habilidades de comunicación del personal de salud involucrado en el proceso de atención, para mejorar la seguridad y la calidad de la atención clínica, así como la gestión de la organización de salud y ayudar a reparar los problemas en la relación médico paciente, contribuyendo a garantizar la satisfacción del paciente ${ }^{(14)}$. Por ello, el objetivo del presente estudio busca analizar las reclamaciones del paciente y sus familiares en el servicio de emergencia adultos del Hospital Nacional Edgardo Rebagliati Martins durante el año 2019.

\section{MÉTODOS}

\section{Diseño}

El presente estudio se realizó mediante un diseño no experimental, tipo descriptivo, prospectivo de corte transversal.

\section{Población y muestra}

La población de estudio estuvo conformada por reclamaciones registradas en el Libro de Reclamaciones en Salud del servicio de emergencia adultos del Hospital Nacional Edgardo Rebagliati Martins. Se obtuvo una muestra no probabilística por conveniencia, conformada por 109 reclamos durante el periodo comprendido entre los meses de mayo a noviembre de 2019; excluyéndose de la muestra las reclamaciones registradas previa y posteriormente al período de estudio, las redactadas de manera confusa o ilegible y las que corresponden a otras instancias diferentes al servicio de emergencia adultos. 


\section{Procedimientos y variables}

Para recolectar la información, se elaboró una ficha de recolección de datos teniendo como base la información del Libro de Reclamaciones en Salud aprobado mediante Decreto Supremo N. ${ }^{\circ} 002-2019-S A$ Reglamento para la Gestión de Reclamos y Denuncias de los Usuarios de las Instituciones Administradoras de Fondos de Aseguramiento en Salud - IAFAS, Instituciones Prestadoras de Servicios de Salud - IPRESS y Unidades de Gestión de Instituciones Prestadoras de Servicios de Salud - UGIPRESS, públicas, privadas o mixtas ${ }^{(15)}$. Los datos consignados en la ficha fueron: fecha de reclamación, nombre, género, tipo de usuario, motivo de reclamación, área donde ocurrió la reclamación, personal aludido y fecha de respuesta del área responsable de la reclamación.

Actualmente, no existe una codificación o taxonomía estándar para las reclamaciones, por ello, se utilizó el método Kawakita Jiro o diagrama de afinidad para estratificar los motivos de reclamaciones, a través de este método cada reclamación fue revisada y asignada a una, dos o más dimensiones, de acuerdo al contenido, obteniéndose nueve dimensiones: desacuerdo del paciente con la atención o tratamiento recibido, demora en la atención, inadecuado trato por parte del personal asistencial, inadecuado trato por parte del personal administrativo, demora en los procesos administrativos, inadecuado entorno, desabastecimiento de recursos, escasa comunicación y otros.

\section{Análisis estadístico}

La validez de contenido de la ficha de recolección de datos se realizó mediante un juicio de expertos, convocándose a seis expertos, obteniendo un coeficiente de Kappa con un resultado de 0,78. La confiabilidad se evaluó mediante el método test retest que implicó la aplicación de la misma ficha dos veces a la misma persona, dejando un intervalo de tiempo entre el test y el retest, obteniéndose el resultado de 0,73 . Para el procesamiento de los datos se utilizó el programa SPSS versión 25 en español para calcular frecuencias absolutas y relativas de las variables del objeto del estudio y se realizó un análisis descriptivo de los datos sociodemográficos.

\section{Consideraciones éticas}

Los datos no fueron utilizados en beneficio propio, se cumplió con los principios básicos de ética. El estudio fue aprobado por la Oficina de Investigación y Docencia, así como por el Comité de Ética del Hospital Nacional Edgardo Rebagliati Martins, asegurándose la respectiva confidencialidad de la información registrada.

\section{RESULTADOS}

En el periodo deestudio, seanalizaron 109reclamaciones registradas en el Libro de Reclamaciones en Salud del servicio de emergencia adultos del Hospital Nacional Edgardo Rebagliati Martins. Los resultados muestran que $54,1 \%$ fueron mujeres y $45,9 \%$ hombres. Se observó que $47,7 \%$ fueron presentadas por familiares directos del paciente. Asimismo, en el análisis de reclamaciones por dimensiones se evidenció que $23,5 \%$ corresponden a inadecuado trato por parte del personal asistencial seguido de $23 \%$ por demora en la atención y $13,9 \%$ relacionada al desacuerdo del paciente con la atención o tratamiento recibido (Figura 1).

Los resultados muestran que, en cada una de las reclamaciones existe más de un motivo que genera la insatisfacción del paciente o familiar, evidenciándose un total de 187 motivos dentro de las 109 reclamaciones analizadas; dependiendo de su naturaleza cada motivo fue asignado a las diferentes dimensiones. Los resultados correspondientes a la dimensión desacuerdo del paciente con la atención o tratamiento recibido, permitieron identificar que $10,2 \%$ de pacientes se encuentran en desacuerdo con la atención recibida; respecto a la dimensión demora en la atención, 12,8\% reclamaron por la falta de atención oportuna; en las dimensiones inadecuado trato por parte del personal asistencial y administrativo, se evidenció que $22 \%$ de reclamaciones se debieron a un trato inadecuado (descortés y grosero) por parte del personal del servicio. En relación a la dimensión escasa comunicación, 7,5\% de los pacientes señalaron que el motivo de insatisfacción se produjo por la mala calidad de la información recibida; en lo que corresponde a la dimensión demora en los procesos administrativos, 8,5\% manifestaron que el motivo de reclamación se debió a procesos administrativos lentos y al procedimiento lento en el área de admisión siendo este último el más descrito en las reclamaciones. Los resultados obtenidos en la dimensión inadecuado entorno, mostraron que 2,1\% de pacientes se encuentran descontentos ante la falta de ambientes confortables; mientras que en la dimensión desabastecimiento de recurso, $1,6 \%$ por la falta de medicamentos en farmacia y en la última dimensión otros, un 0,5\% atribuyeron la reclamación a la falta de especialistas en el servicio de emergencia (Tabla 1).

En el análisis de los 187 motivos de reclamaciones se evidenció que 133 correspondieron a problemas de tipo prestacional, asociados a la atención directa del paciente y 54 de tipo administrativo. Los meses en los que se presentó el mayor número de reclamaciones de tipo prestacional fueron mayo y agosto, siendo estos mismos meses en los cuales las reclamaciones de tipo administrativo se presentaron en mayor cantidad (Figura 2).

En relación al análisis de frecuencia de reclamaciones por áreas del servicio de emergencia adultos del Hospital Nacional Edgardo Rebagliati Martins, se 
observó que $43 \%$ ocurrieron en el área de medicina interna, seguido de $16,1 \%$ en el área triaje y $9,7 \%$ en enfermería (Figura 3).

En la revisión de los casos, el 32,1\% de reclamaciones corresponden a las dimensiones inadecuado trato por parte del personal asistencial y administrativo, de las cuales $66,7 \%$ aluden al personal profesional de la salud: médico $40 \%$ y enfermera $20 \%$ y $33,3 \%$ señalan al personal que ofrece el primer contacto con la institución como vigilante $(15,6 \%)$, admisionista $(6,7 \%)$ y personal de atención al asegurado (4,4\%).
Con respecto al análisis de resolución de reclamaciones medido en días, resulta preciso señalar que la norma establece que el plazo máximo de atención de reclamaciones no debe exceder los 30 días hábiles. El presente estudio permitió evidenciar que, 60,6\% de reclamaciones se resolvieron de manera satisfactoria mediante una explicación o una disculpa; 69,1\% dentro de los primeros 10 días; $19,1 \%$ entre 11-20 días y $11,8 \%$ entre los $21-30$ días. Mientras que $39,4 \%$ de reclamaciones fueron resueltas posterior a los 30 días, sobrepasando el plazo de respuesta que exige la norma vigente (Figura 4).

Tabla 1. Frecuencia de motivos de reclamaciones registradas en el Libro de Reclamaciones en Salud del servicio de emergencia adultos.

\begin{tabular}{|c|c|c|c|c|}
\hline \multicolumn{3}{|r|}{ Motivos de reclamaciones } & $\mathbf{N}$ & $\%$ \\
\hline \multirow{16}{*}{ Motivos prestacionales } & \multirow[t]{4}{*}{ Dimensión } & Desacuerdo del paciente con la atención o tratamiento recibido & 26 & 13,9 \\
\hline & & Desacuerdo con la atención recibida & 19 & 10,2 \\
\hline & & Desacuerdo con el tratamiento recibido & 6 & 3,2 \\
\hline & & Negligencia o mala praxis & 1 & 0,5 \\
\hline & \multirow[t]{4}{*}{ Dimensión } & Demora en la atención & 43 & 23 \\
\hline & & Tiempo de espera prolongado en triaje & 17 & 9,1 \\
\hline & & Falta de atención oportuna & 24 & 12,8 \\
\hline & & Demora en la realización de procedimientos & 2 & 1,1 \\
\hline & \multirow[t]{4}{*}{ Dimensión } & Inadecuado trato por parte del personal asistencial & 44 & 23,5 \\
\hline & & Trato inadecuado (descortés, grosero) & 26 & 13,9 \\
\hline & & Falta de privacidad en la atención & 1 & 0,5 \\
\hline & & Desconsideración por parte del personal /deshumanizado & 17 & 9,1 \\
\hline & \multirow[t]{4}{*}{ Dimensión } & Escasa comunicación & 22 & 11,8 \\
\hline & & Escasa comunicación sobre el estado de salud & 4 & 2,1 \\
\hline & & Información insuficiente a los familiares & 4 & 2,1 \\
\hline & & Mala calidad de la información & 14 & 7,5 \\
\hline \multirow{21}{*}{ Motivos administrativos } & \multirow[t]{3}{*}{ Dimensión } & inadecuado trato por parte del personal administrativo & 16 & 8,6 \\
\hline & & Trato inadecuado (descortés, grosero) & 15 & 8,1 \\
\hline & & Atención discriminatoria & 1 & 0,5 \\
\hline & \multirow[t]{6}{*}{ Dimensión } & demora en los procesos administrativos & 24 & 12,8 \\
\hline & & Procedimientos lentos en admisión & 6 & 3,2 \\
\hline & & Demora en la entrega de medicamentos & 1 & 0,5 \\
\hline & & Procesos administrativos lentos & 10 & 5,3 \\
\hline & & Procesos administrativos no definidos & 3 & 1,6 \\
\hline & & Demora en el traslado a hospitalización & 2 & 1,1 \\
\hline & & Entrega de la cena es muy tarde & 1 & 0,5 \\
\hline & & Incumplimiento de horarios de atención en farmacia & 1 & 0,5 \\
\hline & \multirow[t]{4}{*}{ Dimensión } & inadecuado entorno & 7 & 3,7 \\
\hline & & Ambientes no confortables & 4 & 2,1 \\
\hline & & Falta de privacidad en los ambientes & 2 & 1,1 \\
\hline & & Inadecuada señalización & 1 & 0,5 \\
\hline & \multirow[t]{3}{*}{ Dimensión } & desabastecimiento de recursos & 4 & 2,1 \\
\hline & & Falta de medicamentos en farmacia & 3 & 1,6 \\
\hline & & Falta de silla ruedas & 1 & 0,5 \\
\hline & \multirow[t]{3}{*}{ Dimensión } & otros & 1 & 0,5 \\
\hline & & Falta de especialista & 1 & 0,5 \\
\hline & & Total & 187 & 100 \\
\hline
\end{tabular}




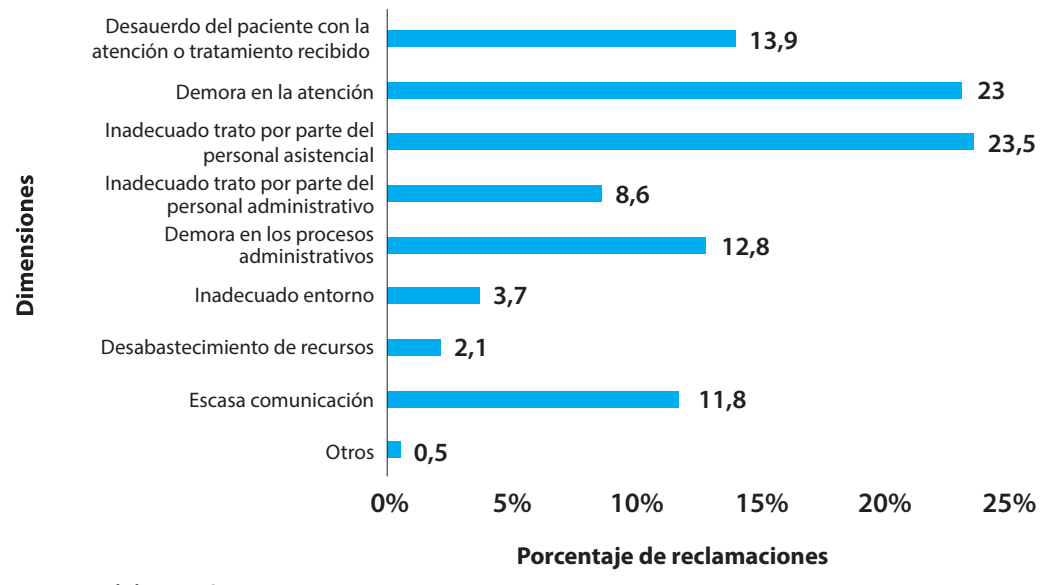

Fuente: Elaboración propia.

Figura 1. Análisis de reclamaciones por dimensiones en el servicio de emergencia adultos.

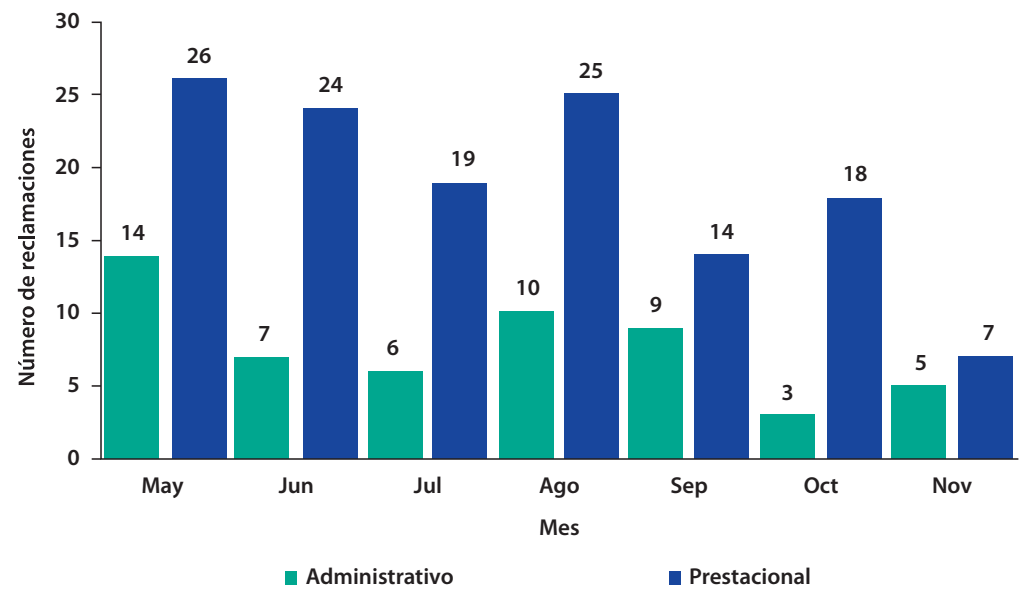

Fuente: Elaboración propia.

Figura 2. Distribución de reclamaciones de tipo prestacional y administrativo por meses en el servicio de emergencia adultos.

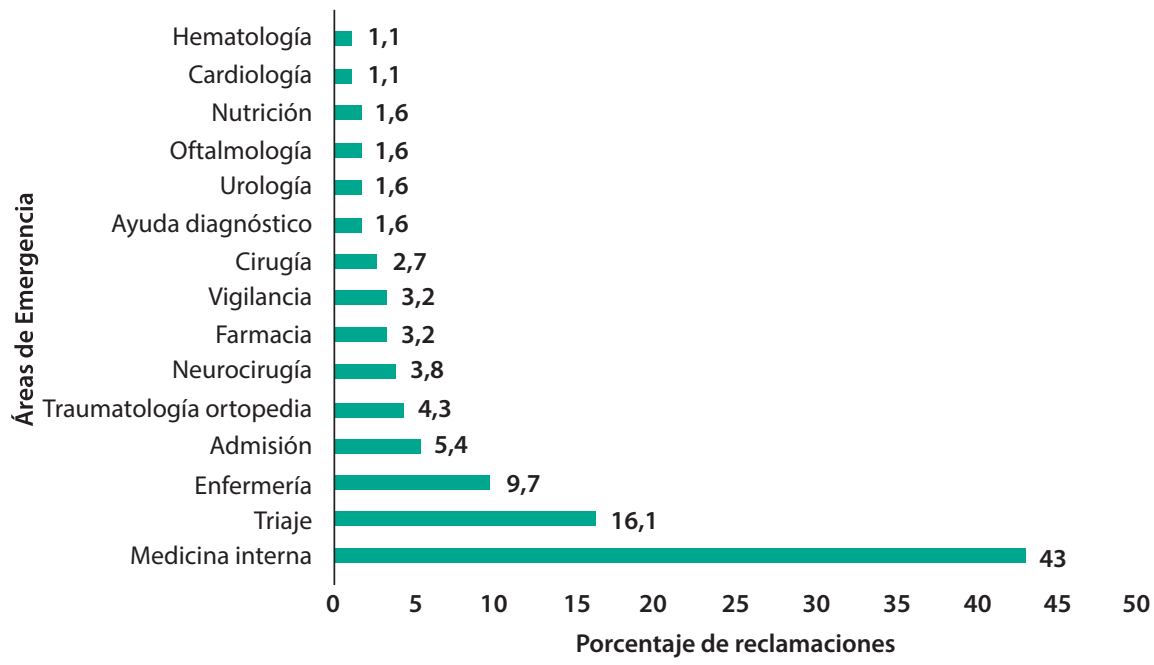

Fuente: Elaboración propia.

Figura 3. Análisis de frecuencia de reclamaciones por áreas del servicio de emergencia adultos. 


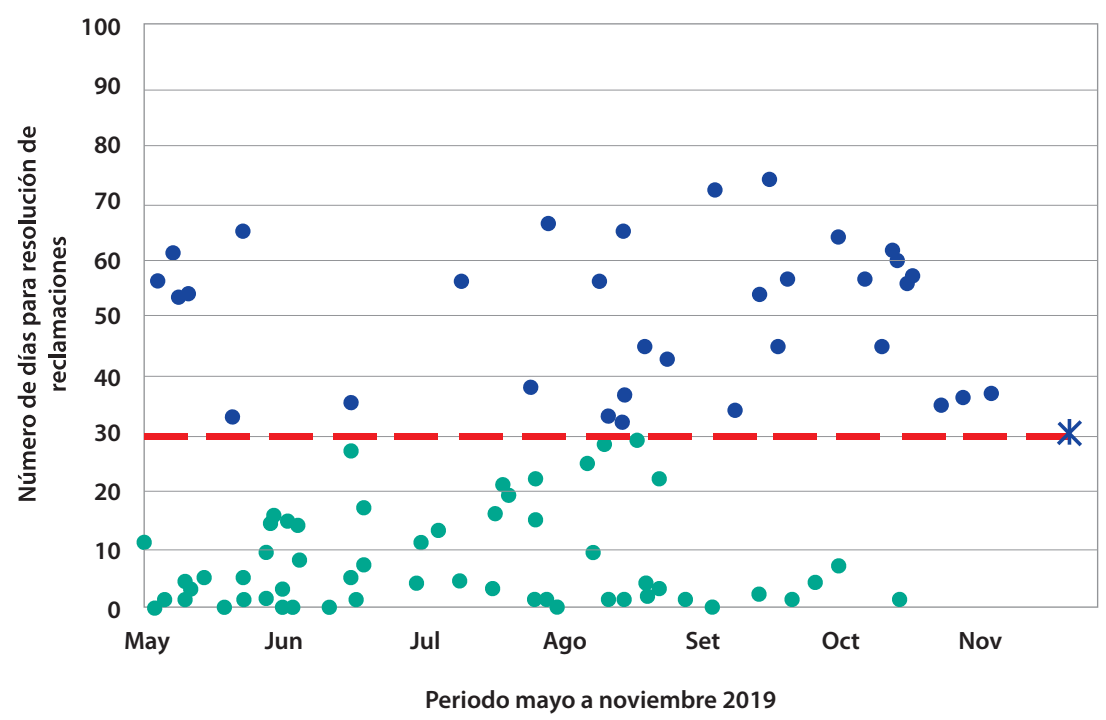

Fuente: Elaboración propia.

Figura 4. Tiempo de resolución de reclamaciones en el servicio de emergencia adultos.

\section{DISCUSIÓN}

La creciente demanda de los servicios de salud es uno de los elementos que ha favorecido la saturación en los servicios de emergencia, esto asociado a la demora en la atención o a la realización de pruebas de ayuda diagnóstica, procedimientos y terapéutica, generan un descenso en los indicadores de calidad como pacientes no atendidos, largos tiempos de espera, estancias prolongadas, incremento de ocurrencia de eventos adversos, insatisfacción de pacientes-familiares y reclamaciones. Asimismo, la saturación de los servicios de emergencia repercute en el personal de salud, manifestado en la sobrecarga laboral que desencadena un trato inadecuado, conflictos interpersonales y ruptura en la relación médico paciente ${ }^{(16,17)}$. Las reclamaciones son el resultado de expectativas no satisfechas del paciente o reflejan la mala calidad del servicio. El análisis permite mostrar la existencia de puntos débiles que se presentan desde la entrada del paciente por triaje hasta el alta del servicio $y$, a su vez sirve, como herramienta para la mejora de la calidad percibida ${ }^{(18)}$.

Los resultados del presente estudio muestran que el inadecuado trato por parte del personal asistencial y la demora en la atención en el servicio de emergencia, continúan siendo los principales motivos de insatisfacción del paciente, reflejados a través de reclamaciones. Estas mismas motivaciones han logrado ser evidenciadas en otra investigación realizada en un hospital en Australia, en la cual señalan la existencia de tres principales motivos de reclamaciones como, el inadecuado tratamiento y diagnóstico 16,9\%, mala actitud o trato descortés $13 \%$ y demora en el tratamiento por tiempos de espera prolongados $11,9 \%$, lo cual termina afectando la satisfacción del paciente y familiares, la comunicación y las relaciones interpersonales ${ }^{(19,20)}$.

La condición crítica del paciente que acude al servicio de emergencia debería generar en los profesionales de salud que intervienen a lo largo del proceso de atención, la necesidad de sentir preocupación, empatía y respeto por el paciente ${ }^{(21)}$. Si bien es cierto, lo señalado anteriormente debería formar parte de la idiosincrasia del personal del salud, el análisis de reclamaciones del estudio de investigación realizado determinó que, en muchos casos, coexiste más de un problema que motiva la inconformidad del paciente, siendo el principal el trato inadecuado, descortés y grosero por parte del personal asistencial, asociado especialmente a la atención médica y de enfermería, hecho que sigue siendo un problema en los hospitales a cargo del Estado. De la misma manera, estos hallazgos han sido evidenciados en otros estudios, en los cuales, la percepción de los familiares frente a la atención médica recibida resulta ser desfavorable, reafirmando la necesidad del paciente y de sus familiares de recibir un trato digno. Asimismo, investigaciones realizadas en México a través de encuestas, demostraron que la atención recibida por parte del personal de salud obtuvo resultados preocupantes, en las cuales, solo un grupo pequeño de pacientes encuestados expresaron que fueron tratados con dignidad, mientras que más de la mitad refirieron que sus derechos como personas nunca fueron respetados ${ }^{(22,23)}$.

Actualmente, los establecimientos de salud trabajan de manera ardua en la difusión de los deberes y derechos en salud de los pacientes, condición fundamental para que cualquier persona pueda gozar de una atención oportuna en casos de emergencia; en un medio ambiente que reúna las condiciones adecuadas de 
infraestructura y de recursos mínimos indispensables; situación que no viene siendo cumplida en los hospitales públicos del sector Salud; por ello, en el presente estudio, la falta de oportunidad en la atención se convirtió en el segundo problema evidenciado por los pacientes y sus familiares, manifestado a través de las reclamaciones. Cabe enfatizar que este resultado no solo corresponde a estudios realizados en nuestro medio sino también han sido demostrados en otra investigación realizada en un hospital de España, en la cual, la demora y la atención no oportuna constituyeron la cuarta causa de insatisfacción del paciente debido a dos razones, primero porque las expectativas del paciente no fueron satisfechas y segundo porque el problema de salud por el cual acudieron al servicio de emergencia no fue solucionado de manera inmediata(24).

Según Saizar(25), el creciente acceso a la información impulsa al paciente a permanecer informado acerca del proceso de su enfermedad, diagnóstico y terapéutica, por otro lado, la saturación del servicio de emergencia ha generado atenciones poco personalizadas, carentes de información y comunicación, haciendo sentir al paciente como si no fuera escuchado y que los profesionales de salud no comprenden la experiencia del dolor desde la perspectiva del enfermo. La unión de estas dos razones genera en el paciente cuestionamientos acerca del proceso de atención o terapéutica recibida, produciéndose conflictos o denuncias por desacuerdo con los profesionales de la salud; asimismo, Mack et al. ${ }^{(26)}$ refieren que, en cuestiones de salud, el paciente y sus familiares están priorizando dos temas que definen una adecuada atención en salud que son, la relación médico paciente y la comunicación e información que les brinda el médico en relación a su estado de salud. Bajo esa misma perspectiva, los resultados del estudio de investigación realizado, evidenciaron como tercer motivo de reclamación, el desacuerdo del paciente con la atención o terapéutica recibida en la emergencia que desencadena una ruptura en la relación médico paciente y en algunos casos puede llegar hasta denuncias por presunta negligencia médica o mala praxis.

Por lo señalado anteriormente, resulta importante enfatizar que la comunicación e información médica que se brinda al paciente y sus familiares se convierte en un factor determinante de los resultados de la calidad asistencial. La comunicación entre el médico, paciente y familiares debe ser adecuada, oportuna y comprensible, contribuyendo a brindar una información clara del estado de salud, tratamiento, procedimientos, evolución y pronóstico de la enfermedad ${ }^{(27)}$. En atención a lo descrito, la presente investigación permitió evidenciar otro motivo importante de reclamación como es la escasa comunicación sobre el estado de salud del paciente, así como la insuficiente información brindada a los familiares y la mala calidad de la información, lo cual, en muchos casos, termina deteriorando la relación médico paciente. Por ello, resulta importante tener en cuenta, que un estudio similar realizado en áreas críticas dio a conocer que un gran número de pacientes calificaron como mala la explicación dada por el médico sobre la causa de la enfermedad y de la misma forma, calificaron como deficiente la explicación recibida sobre el proceso de recuperación de la enfermedad y la información brindada por el médico referente a la urgencia de recibir un medicamento o procedimiento con la autorización del paciente, ratificándose así que las dificultades que surgen durante el proceso de comunicación entre el paciente y los profesionales de salud, continua siendo una problemática que afecta a varios establecimientos de salud ${ }^{(28)}$.

En cuanto al tiempo de resolución de reclamaciones cabe precisar que las entidades públicas se encuentran obligadas, según norma, a dar respuesta a las reclamaciones en un plazo máximo que no exceda los 30 días hábiles, a través de medios físicos o electrónicos. El presente estudio mostró que un gran número de reclamaciones fueron resueltas de manera satisfactoria dentro del periodo establecido. De la misma forma, investigaciones similares demuestran que las reclamaciones presentadas por el paciente y sus familiares son resueltas dentro del periodo establecido por la norma vigente, lo cual, permite a los establecimientos de salud generar oportunidades que contribuyan a mejorar la calidad en la atención y a su vez, evitar que sucesos similares vuelvan a presentarse, para lo cual deberán establecer medidas correctivas ${ }^{(29,30)}$.

Durante el estudio de investigación se presentaron ciertas limitaciones como un tamaño de muestra relativamente pequeño. Las reclamaciones en su gran mayoría resultan complejas y en algunos casos pueden haber sido clasificadas de manera incorrecta, lo que conlleva a un sesgo de medición. Los motivos de reclamaciones fueron clasificados revisando caso por caso; sin embargo, al no existir una taxonomía o clasificación estándar, se dificulta la comparación con otros estudios a nivel nacional e internacional.

\section{CONCLUSIÓN}

Se evidenció que un gran número de reclamaciones fueron presentadas por los familiares directos del paciente. En su mayoría, los reclamos fueron de tipo prestacional, asociados a la atención directa del paciente, principalmente debido a problemas por el trato recibido por el personal médico y enfermera y por la demora en la atención. El área de medicina interna obtuvo la mayor cantidad de reclamaciones. La mayoría de reclamaciones se resolvieron de manera satisfactoria mediante una disculpa o explicación, quedando una menor proporción de reclamaciones resueltas fuera de los plazos establecidos en la norma. 
Declaración: Se declara que el material contenido en el manuscrito no ha sido publicado previamente ni remitido a otra revista biomédica.

Contribuciones de autoría: Los autores participaron en la génesis de la idea, diseño del proyecto, recolección e interpretación de datos, análisis de resultados y preparación del manuscrito del presente trabajo de investigación.
Financiamiento: Autofinanciado por los autores.

Conflicto de interés: Los autores declaran no tener conflicto de interés en la publicación de este artículo.

Recibido: 22 de octubre del 2019

Aprobado: 17 de marzo del 2020
Correspondencia: Isabel J. Alamo Palomino.

Dirección: Av. San Felipe 1161. Dpto 401. Jesús María, Lima-Perú.

Teléfono: 958934984

Correo: Isabel.alamo7@gmail.com

\section{REFERENCIAS BIBLIOGRÁFICAS}

1. Bongale S, Young I. Why people complain after attending emergency departments. Emerg Nurse. 2013; 21(6):26-30. doi: 10.7748/en2013.10.21.6.26 e1200.

2. Matzumura J, Gutiérrez H, Zamudio L. Satisfacción de la atención en el servicio de emergencia de una institución privada de salud. Qhalikay Revista de Ciencias de la Salud 2018; 2(1): 1-11. doi: 10.33936/qhalikay.v2i1.1401.

3. Salazar R, Gutiérrez H, Zamudio, L. Conocimiento sobre derechos en salud de padres con niños sometidos a cirugía en un hospital especializado Perú, 2016. In Crescendo. 2018; 9(4): 667-79. doi: 10.21895/incres.

4. Añel-Rodriguez RM, Cambero-Serrano MI, Irurzun-Zuazabal E. Análisis de las reclamaciones de pacientes en atención primaria: una oportunidad para mejorar la seguridad clínica. Rev Calid Asist. 2015; 30(5): 220-25. doi: 10.1016/j. cali.2015.04.007

5. Shimabuku R, Fernández D, Carlos G, Granados K, Maldonado R, Nakachi $G$. Las reclamaciones de los usuarios externos en un centro pediátricos de referencia nacional en Lima, Perú. An Fac med. 2014; 75(3): 245-50. doi: 10.15381/anales.v75i3.9779.

6. Reader TW, Gillespie A, Roberts J. Patient complaints in healthcare systems: a systematic review and coding taxonomy. BMJ Qual Saf. 2014; 23(8): 678-89. doi: 10.1136/bmjqs-2013-002437.

7. Friele RD, Reitsma PM, de Jong JD. Complaint handling in healthcare: expectation gaps between physicians and the public; results of a survey study. BMC Res Notes. 2015; 8(1): 529-36. doi: 10.1186/s13104-015-1479-z.

8. Kroening HL, Kerr B, Bruce J, Yardley I. Patient complaints as predictors of patient safety incidents. Patient Exp J. 2015; 2(1): 94-101. doi: 10.35680/2372 0247.1052 .

9. Harrison R, Walton M, Healy J, Smith-Merry J, Hobbs C. Patient complaint about hospital services: applying a complaint taxonomy to analyse and respond to complaints. Int J Qual Health Care. 2016; 28(2): 240-45 doi: 10.1093/ intqhc/mzw003.

10. Veneau L, Chariot P. How do hospitals handle patients complaints? An overview from the Paris area. J Forensic Leg Med. 2013; 20(4): 242-47. doi: 10.1016/j.jflm.2012.09.013

11. Gallagher TH, Levinson W. Physicians with multiple patient complaints: ending our silence. BMJ Qual Saf. 2013; 22(7): 521-24. doi: 10.1136/ bmjqs-2013-001880.

12. Bouwman $R$, Bomhoff $M$, Robben $P$, Friele R. Patients' perspectives on the role of their complaints in the regulatory process. Health Expect. 2016; 19(2): 483-96. doi: 10.1111/hex.12373.

13. Schaad B, Bourquin C, Bornet F, Currat TH, Saraga M, Panese F, et al. Dissatisfaction of hospital patients, their relatives, and friends: Analysis of accounts collected in a complaints center. Patient Educ Couns. 2015; 98(6): 771-76. doi: 10.1016/j.pec.2015.02.019.

14. Bismark MM, Spittal MJ, Gurrin LC, Ward M, Studdert DM. Identification of doctors at risk of recurrent complaints: a national study of healthcare complaints in Australia. BMJ Oual Saf. 2013; 22(7): 532-40. doi: 10.1136/ bmjqs-2012-001691.

15. Reglamento para la Atención de Reclamos y Denuncias de los usuarios de las Instituciones Administradoras de Fondos de Aseguramiento en Salud - IAFAS, Instituciones Prestadoras de Servicios de Salud - IPRESS y Unidades de Gestión de Instituciones Prestadoras de Servicios de Salud - UGIPRESS, públicas, privadas o mixtas. D.S. N 002-2019-SA (30 de enero de 2019). Lima:
Ministerio de Salud.

16. Tudela P, Modol JM. La saturación en los servicios de urgencias hospitalarios. Emergencias. 2015; 27(2): 113-20.

17. Catron TF, Guillamondegui OD, Karras J, CooperWO, Martin BJ, Dmochowski RR, et al. Patient complaints and adverse surgical outcomes. Am J Med Qual. 2016; 31(5): 415-22. doi: 10.1177/1062860615584158.

18. García-García A, Arévalo A, García-lglesias MA, Sánchez M, DelgadoVicente MA, Bajo A, et al. Información, comunicación y trato en un servicio de urgencias: evaluación mediante una encuesta de opinión. Rev Calid Asist. 2015; 30(4): 203-9. doi: 10.1016/j.cali.2015.04.005

19. Taylor DM, Wolfe R, Cameron PA. Complaints from emergency department patients largely result from treatment and communication problems. Emerg Med. 2002; 14(1): 43-9. doi: 10.1046/j.1442-2026.2002.00284.x.

20. García R, Gálvez N. Calidad de atención asociada a la satisfacción de usuario externo en los establecimientos asistenciales de salud: Minsa-Essalud, Chongoyape-Perú. Revista Tzhoecoen. 2016; 8(2): 1-10.

21. Canchero A, Matzumura J, Gutierrez H. Satisfacción del familiar del paciente en la unidad de cuidados intensivos del Hospital Nacional Dos de Mayo, 2018. An Fac med. 2019; 80(2): 177-82. doi: 10.15381/anales.802.16412.

22. Crespo MI, Pérez J, Gonzales MP, Ballesteros AM. Más allá de la hoja de reclamaciones. Análisis cualitativo de las reclamaciones y sugerencias en atención primaria. Revista Enfermería CyL. 2019; 11(1): 5-11.

23. Zamora S. Satisfacción sobre la infraestructura y la calidad de atención en la consulta gineco-obstetricia de un establecimiento de atención primaria. Horiz Méd. 2016; 16(1): 38-47.

24. Pérez DC, Gil MA, Abreu P. Estudio de las reclamaciones de los usuarios dirigidas al Servicio de Oftalmología, en el área del Complejo Hospitalario Ntra. Sra. De la Candelaria entre los años 2009 y 2013. Archivos de la Sociedad Canaria de Oftalmología. 2014; 25: 26-30.

25. Saizar, M. Motivos de encuentro y desencuentro en la relación entre profesionales y usuarios del sistema de salud público de Argentina. Trabajo Social. 2015; 17: 95-112.

26. Mack JW, Jacobson J, Frank D, Cronin AM, Horvath K, Allen V, et al. Evaluation of patient and family outpatient complaints as a strategy to prioritize efforts to improve cancer care delivery. Jt Comm J Qual Patient Saf. 2017; 43(10): 498507. doi: $10.1016 /$ j.jcjq.2017.04.008

27. Liang C, Gu D, Tao F, Jain H, Zhao Y, Ding B. Influence of mechanism of patient-accessible hospital information system implementation on doctor-patient relationships: A service fairness perspective. Information \& Management. 2017; 54(1): 57-72. doi: 10.1016/j.im.2016.03.010.

28. Cehua E, Matzumura J, Gutierrez H Zamudio L. Calidad de la información médica brindada a las pacientes atendidas en el servicio de emergencia de ginecología y obstetricia de un hospital categoría II-2 en Lima, Perú. REVCOG. 2017; 22(2): 31-7.

29. Bustamante Z, Becerra N. Reclamaciones de los usuarios de un hospital de tercer nivel de Lima-Perú 2015. Revista Peruana de Obstetricia y Enfermería. 2016; 12(1): 10p.

30. Salvador S, Millán J, Téllez C, Pérez C, Oliver C. Implantación de un plan de calidad en un servicio de urgencias y su impacto en la satisfacción de paciente. Emergencias. 2013; 25(3): 163-70. 